\title{
Novel Method Employing Accelerated-Oscillated Wave Saline Solutions to Unblock Blood Vessels-Physics and Fluid Dynamics Perspectives and Simulations
}

\author{
Stephon Stewart, Dineshen Chuckravanen* \\ British Association of Cognitive Neuroscience (BACN), Department of Psychology, University of Essex, Colchester, UK \\ Email: *dineshen2013@gmail.com
}

How to cite this paper: Stewart, S. and Chuckravanen, D. (2021) Novel Method Employing Accelerated-Oscillated Wave Saline Solutions to Unblock Blood Vessels-Physics and Fluid Dynamics Perspectives and Simulations. Open Journal of Biophysics, 11, 415424.

https://doi.org/10.4236/ojbiphy.2021.114018

Received: August 17, 2021

Accepted: October 26, 2021

Published: October 29, 2021

Copyright ( 2021 by author(s) and Scientific Research Publishing Inc. This work is licensed under the Creative Commons Attribution International License (CC BY 4.0).

http://creativecommons.org/licenses/by/4.0/ (c) (i) Open Access

\begin{abstract}
This research assesses the speed of blood flow across blood vessels and more specifically the veins in terms of Reynold's number (laminar flow vs. turbulence flow) and in terms of overall speed of the blood when being injected with high-speed saline particles. The authors propose a novel technique to generate accelerated-waved particles built from saline solution to enable the unblocking of partially-blocked healthy-walled veins, and to restore normal operations of these veins. The novel technique encompasses a pump that accelerates saline solutions into the blood stream of the vein and these oscillated waves break down the fats or deposits inside the veins in order to help the blood to flow freely without any obstruction. This research simulated the vein with blood stream using characteristics of the vein in terms of vein diameter, blood density, venous blood flow, and the viscosity of the blood at the normal body temperature. The speed of the overall blood flow after the injection of the accelerated saline droplet solution was determined as well as the depth of penetration of the accelerated particles in order to cleanse the inside of the vein. Results are promising in terms of not altering significantly the overall speed of the bloodstream and also in terms of efficacy of the length of the vein which is being cleaned using this accelerated particle method.
\end{abstract}

\section{Keywords}

Arteries, Mathematical Models and Simulations, Sound-Waved Particle Fluids, Unblocking Blood Vessels, Veins

\section{Introduction}

The concept which is proposed here for the unblocking of blood vessels (such as 
veins and arteries) that are hindered by fat deposits or small clots that are found inside the blood vessels is analogous to the working of sound waves in the inner ear. Sound-waved fluids or solutions with $\mathrm{pH}$ similar to blood or saline solution will be injected/cathetered into the bloodstream to create ripples or waves for unblocking blood vessels. Generally speaking, the normal saline solution is a mixture of water and salt and its salt concentration resembles that of tears, blood and other body fluids ( $0.9 \%$ saline) and it is also referred to as an isotonic solution. Therefore, a normal saline solution is considered soothing and friendly to the human body [1]. In order to explain the concept which is used in this research, we will explain in detail how the employed concept in this research mimics the concept of the working of the inner ear.

\subsection{Concept Analogous to Working in Inner Ear}

Hearing relies on a series of complicated stages that transform sound waves in the air into electrical signals. The auditory never transports these signals to the brain subsequently. The sound waves enter the outer ear and then travel along the ear canal leading to the eardrum. The latter then vibrates based on the sound waves that are incident on the eardrum and consequently send these vibrations to tiny bones in the middle ear that are the 1) malleus; 2) incus; and 3) stapes. These bones amplify the sound vibrations and then these are sent to the cochlea which is snail-shaped structure full with fluids in the inner ear. The vibrations cause the fluid inside the cochlea to ripple (vibrate) which produces a travelling wave along the basilar membrane. Hair cells that are found on top of the basilar membrane ride the wave. Sensory cells (hair cells) that are near the wide end of the cochlea detect high-frequency sounds and those that are closer to the center detect low-frequency sounds [2] [3] [4] [5].

Figure 1 demonstrates that the fluid in the top-most tube is set in motion by the application of the piston-like movements of the stapes on the oval window (Left of the figure). The vibrations travel into the fluid of the upper tube of the cochlea and around the tip of the organ into the fluid of the lower tube. One can clearly observe the ripple which is formed in this particular part of the cochlea region.

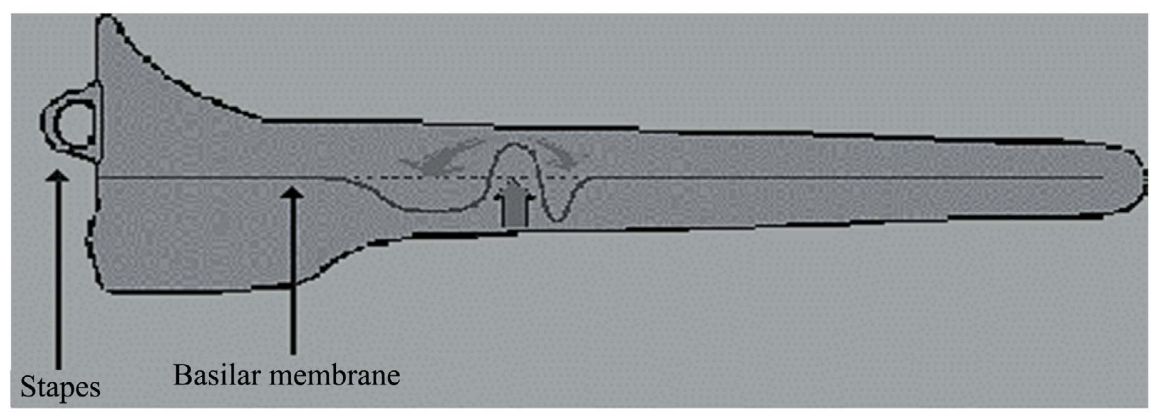

Figure 1. Ripple wave in the fluid of the upper tube of the cochlea owing to vibrations of sound waves. 
Therefore, similar ripple like waves will be generated such that these waves are accelerated inside the blood vessels to unblock clots or fats stuck on the inner wall of the veins. Next, the general characteristics of the blood vessels are described.

\subsection{General Characteristics of Blood Vessels}

The arterial blood flow velocities are in the region of 4.9 to $19 \mathrm{~cm} / \mathrm{s}$ while the venous blood flow ranges from 1.5 to $7.1 \mathrm{~cm} / \mathrm{s}$. The diameters of the blood vessels range from $800 \mu \mathrm{m}$ to $1.8 \mathrm{~mm}$. The blood flow rate in arteries ranges from 3.0 to $26 \mathrm{ml} /$ minute while the blood flow rate in veins ranges from 1.2 to $4.8 \mathrm{ml} /$ minute. The blood flow velocity is the fastest in the middle of the vessel and slowest at the vessel wall owing to friction. Moreover, as viscosity decreases, blood flow increases and so does an increase in perfusion. The resistance to blood flow is inversely proportional to the $4^{\text {th }}$ power of the radius of the blood vessel.

\section{Methodology}

Before elaborating the simulation part, it is crucial to be aware of the following technical terms in terms of types of blood flow in the blood vessels.

\subsection{Laminar Flow}

Laminar flow is shown by the smooth streamlines (see Figure 2) and highly ordered motion and if the blood vessel is long as compared to the entry length then the entrance effects are negligible and the flow is fully developed. The laminar flow happens when the fluid flows in parallel layers without mixing. The velocity of the fluid is constant at any given moment. As the flow is constant, there is no acceleration. The flow is laminar for cylindrical pipes when the Reynolds number is about less than 2500 .

\subsection{Transition from Laminar Flow to Turbulent Flow}

The change from laminar to turbulent flow is not too sudden, it normally occurs over a certain region in which the turbulent flow in the center of the blood vessel

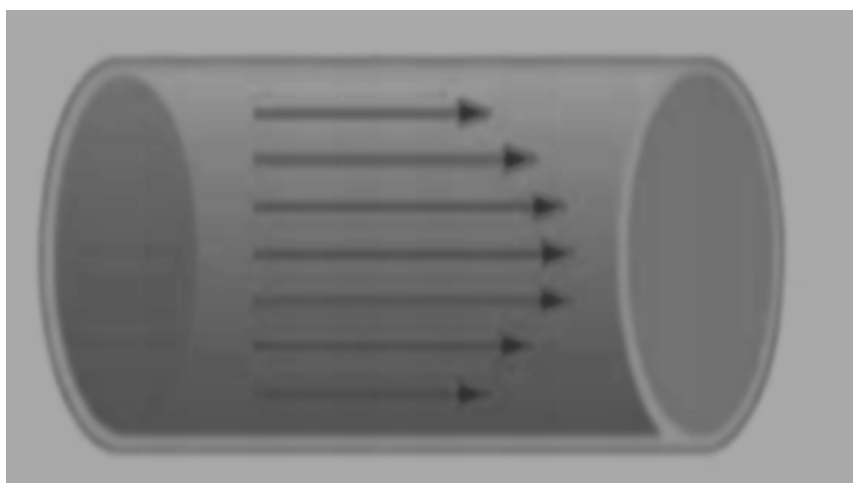

Figure 2. Laminar flow of blood stream in blood vessel. 
and the laminar flow is near the edges of the pipe. The blood flow oscillates between laminar and turbulent flows before it becomes totally turbulent. The flow is transitional when the Reynolds number lies in-between 2300 and 4000.

\section{Turbulent Flow in Blood Vessel}

Turbulent flow occurs by velocity oscillations and highly disordered motion. Turbulent flow happens when streamlines of the liquid are irregular and change over time (see Figure 3). The path of the fluid flow is irregular and form tiny whirlpool regions and the blood flow is turbulent when Reynolds number is greater than 4000. The Reynolds number can reveal whether flow is laminar or turbulent. The transition from laminar to turbulent flow depends on the surface roughness, flow velocity, geometry, surface temperature, and type of fluid.

The flow regime relies on the ratio of inertial forces to viscous forces in the fluid. This ratio is called the Reynolds number and is expressed as:

$R e=$ Inertial Force/Viscous Force:

$$
R e=\frac{\rho V_{\text {avg }} D}{\mu}=\frac{V_{\text {avg }} D}{\mathcal{V}}
$$

In this equation, $\rho$ represents the density of the fluid (SI units: $\left.\mathrm{kg} / \mathrm{m}^{3}\right), V_{\text {avg }}$ represents the average flow speed $(\mathrm{m} / \mathrm{s}), D$ is the diameter of the tube $(\mathrm{m}), \mu$ represents the dynamic viscosity of the fluid (Pa.s or $\mathrm{N} \cdot \mathrm{s} / \mathrm{m}^{2} \mathrm{or} \mathrm{kg} /(\mathrm{m} \cdot \mathrm{s})$ ) and $\mathcal{V}$ denotes the kinematic viscosity of the fluid $\left(\mathrm{m}^{2} / \mathrm{s}\right)$. The critical Reynolds number $R e$ is the number at which the flow becomes turbulent.

\section{Experiments/Simulations}

All simulations were conducted using Matlab software R2021 and a computer of the following properties (i7-7820HQ CPU @ $2.90 \mathrm{GHz}$ and RAM memory 16 GB).

\section{Simulation 1}

The velocity profile of the bloodstream for a determined Reynold number is based on the average velocity of the blood flow in the vein (blood vessel). In order to do the simulation of the behavior of the vein, we have to consider the general characteristics of the vein for an adult (human). The following information relate to the specific features of the human vein and these are outlined as follows [6] [7] [8].

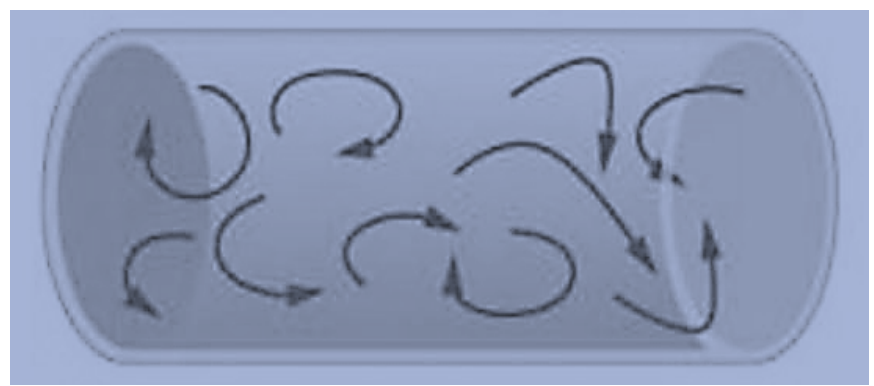

Figure 3. Turbulent flow of blood stream in blood vessel. 
- Blood density $=1060 \mathrm{~kg} / \mathrm{m}^{3}$

- Vein's diameter $=2 \mathrm{~cm}$ (For instance, the average inferior vena cava diameter for an adult is around $2 \mathrm{~cm}(0.78 \mathrm{in}))$

- Venous blood flow $=7.0 \mathrm{~cm} / \mathrm{s}$

- Viscosity of blood $=4.0 \mathrm{cp}$ (ranges from 3.5 to $5.5 \mathrm{cp}$ for normal)

- At normal level, in pascal-second (Pa.s), the viscosity of blood at $37^{\circ} \mathrm{C}$ is normally $3 \times 10^{-3}$ to $4 \times 10^{-3}$ [8], respectively 3 to 4 centipoise $(\mathrm{cP})$ in the centimetre gram second system of units.

- $\mu=(3$ to 4$) \times 10^{-3} \mathrm{~Pa} \cdot \mathrm{s} \leftarrow$

- $V=\mu / \rho=\left\{(3\right.$ to 4$\left.) \times 10^{-3} / 1.06 \times 10^{3}\right\}=(2.8$ to 3.8$) \times 10^{-6} \mathrm{~m}^{2} / \mathrm{s} \leftarrow$

The Reynold's number was determined by the Matlab software to be $397.5 \mathbf{R e}$ (see Figure 4 and Figure 5) and it differs owing to speed of the blood flow and these depend on the chosen characteristics as mentioned before.

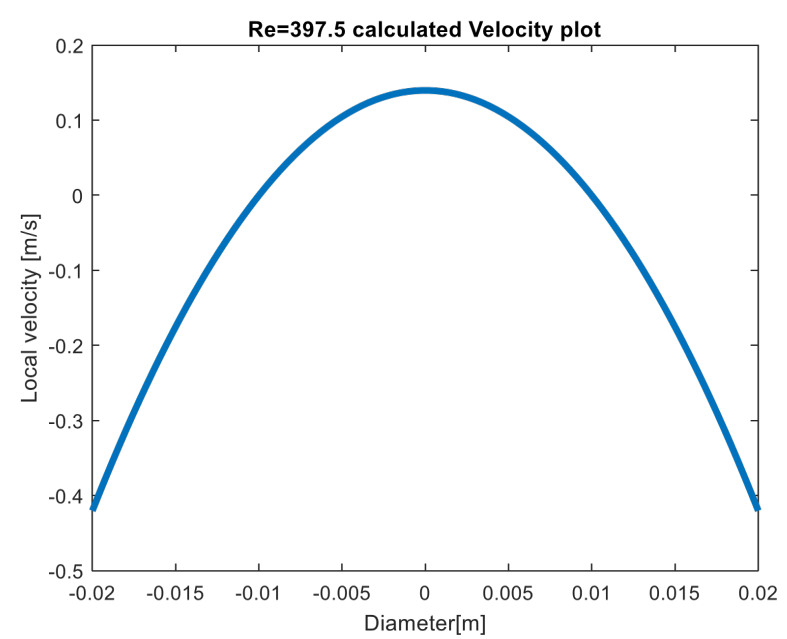

Figure 4. The velocity of the blood stream which is locally in the blood vessel with Reynold's number being 397.5.

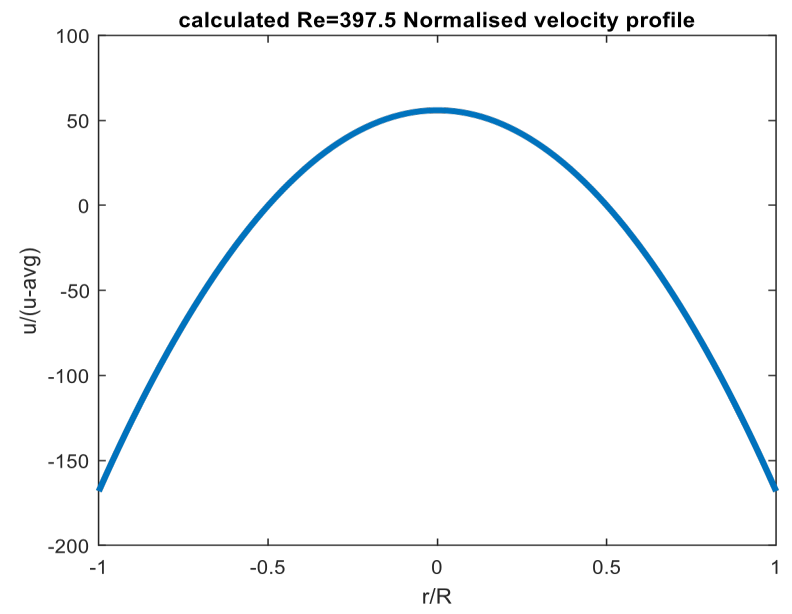

Figure 5. The normalized velocity profile of the blood stream across the blood vessel where $R$ is the radius of the blood vessel and $r$ is the distance from the mid-point of the blood vessel. 


\section{Simulation 2}

For the second simulation, particle accelerated fluid by injection passes through the blood vessel (vein). The sketch below represents the oscillating pump which pumps the saline solution.

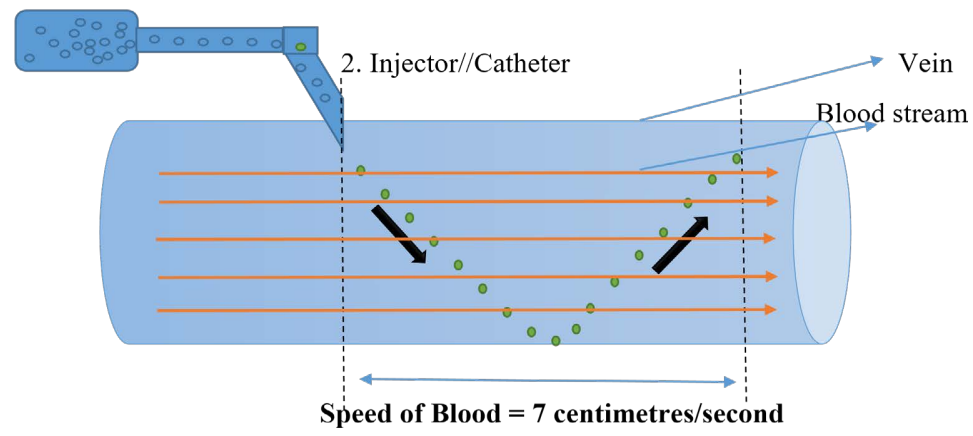

Let us assume that the blood flow is $7.0 \mathrm{~cm}$ per second in the vein, the diameter of the vein is $2 \mathrm{~cm}$ and the volume of blood flow per second is calculated using the volume of cylinder equation:

\section{Step 1: Volume of blood flow per second}

Volume of cylinder $=\pi r^{2} h$

Radius $(r)=2 /(2 \times 100)$ metres

Height $(h)=7 / 100$ metres

Volume of Cylinder $=$ Volume of blood flow per second $=2.1991 \mathrm{e}-05 \mathrm{~m}^{3}$

Step 2: Mass of blood per second

Using the density of blood, one can compute the mass of blood using the following equation:

Let the Mass of blood be $M_{b}$, the density of blood be $\rho_{b}$ and the volume of blood be $V_{b}$.

$$
\frac{\text { Mass of blood }}{\text { Volume of blood }}=\text { Density of blood }
$$

Assuming that the density of blood is $1060 \mathrm{~kg} \cdot \mathrm{m}^{-3}$

$$
\begin{gathered}
M_{b}=\rho_{b} \times V_{b} \\
M_{b}=1060 \times V_{b} \\
M_{b}=1060 \times 2.1991 \mathrm{e}-05 \\
M_{b}=0.0233 \mathrm{~kg}
\end{gathered}
$$

Step 3: Determine the number of oscillated fluid wave particles per centimeter

Let us assume that the shape of the accelerated particle is spherical and therefore the volume of sphere is $\frac{4 \pi r^{3}}{3}$ [9].

Now let us use a syringe tip of $0.3 \mathrm{~mm}$ and therefore the particle emanated from this tip will have a maximum diameter of $0.3 \mathrm{~mm}$.

Thus the volume of 1 particle-accelerated fluid: 


$$
\frac{4 \pi(0.3 / 2000)^{3}}{3}=1.4137 \times 10^{-11} \mathrm{~m}^{3} \leftarrow
$$

\section{Step 4: Using a pump frequency of $300 \mathrm{~Hz}$ to find mass of particles}

In this case scenario, we will be using a pump frequency of $300 \mathrm{~Hz}$ (300 samples per second which insinuates that 300 accelerated particles of saline fluids will be injected per second in the vein and also the saline solution has the same density of blood which is $1060 \mathrm{~kg} / \mathrm{m}^{3}$ [10].

Mass of injected particles $\left(M_{p}\right)=$ Total Volume of particles (per centimetre) $\times$ Density of the particle

$M_{p}=$ Number of particles injected per centimetre $\times 1060$

$$
\begin{gathered}
M_{p}=300 \times 1.4137 \times 10^{-11} \times 1060 \\
M_{p}=4.4956 \times 10^{-6} \mathrm{~kg} \leftarrow
\end{gathered}
$$

Step 5: Conservation of momentum to determine overall speed of the blood stream bombarded with the accelerated particle fluids [11]

$$
\begin{gathered}
M_{b} V_{b}+M_{p} V_{p}=M_{\text {total }} * V_{\text {average }} \\
V_{\text {average }}=\left((0.0233 \times 7.0 \mathrm{~cm} / \mathrm{s})+\left(4.4956 \times 10^{-6} \times 300\right)\right) /(0.0233+4.4956 \mathrm{e}-6) \\
V_{\text {average }}=7.0565 \mathrm{~cm} / \mathrm{s}
\end{gathered}
$$

Therefore, the speed of the total mass of blood and the accelerated particles of saline solution is $7.0565 \mathrm{~cm} / \mathrm{s}$.

Now, the next last step is to find how far this saline-blood stream will move across the vein until it reaches again its original blood stream of $7.0 \mathrm{~cm} / \mathrm{s}$ so that one can quantify the life span of the particles in performing their duties of de-blocking partially blocked veins. In order to determine this, we will resort to kinematic motion.

Step 6: Use of kinematic motion to find how far these accelerated particles can maintain this speed (Step 5)

Using the famous equation of $V^{2}=U^{2}+2$ as where $V$ is the final velocity of an object, $U$ is the initial velocity of an object, $a$ is the acceleration of the object and $s$ is the displacement and we need to find a value for displacement $s$ in metres [12].

$$
\begin{gathered}
\text { Displacement }(s)=\left(V^{2}-U^{2}\right) / 2 a=\left(7.0565^{2}-7.0^{2}\right) / 2 a \\
\text { Acceleration }(a)=\left(V_{2}-V_{1}\right) / \text { time }=(7.0565-7) /(1 \text { second })=0.0565 \mathrm{~cm} / \mathrm{s}^{2} \\
\text { Displacement }(s)=\left(7.0565^{2}-7.0^{2}\right) / 2 \times 0.0565=7.0283 \mathrm{~cm}
\end{gathered}
$$

So this means that the accelerated fluid particles can perform the un-blocking task for a distance of about $7.03 \mathrm{~cm}$.

Simulation: Varying the pump frequency and its effect of the depth of penetration of the accelerated particle saline solution

Figure 6 shows that when the pump frequency is increased, so does the penetration depth of the accelerated particles into the blood vessels but care should 


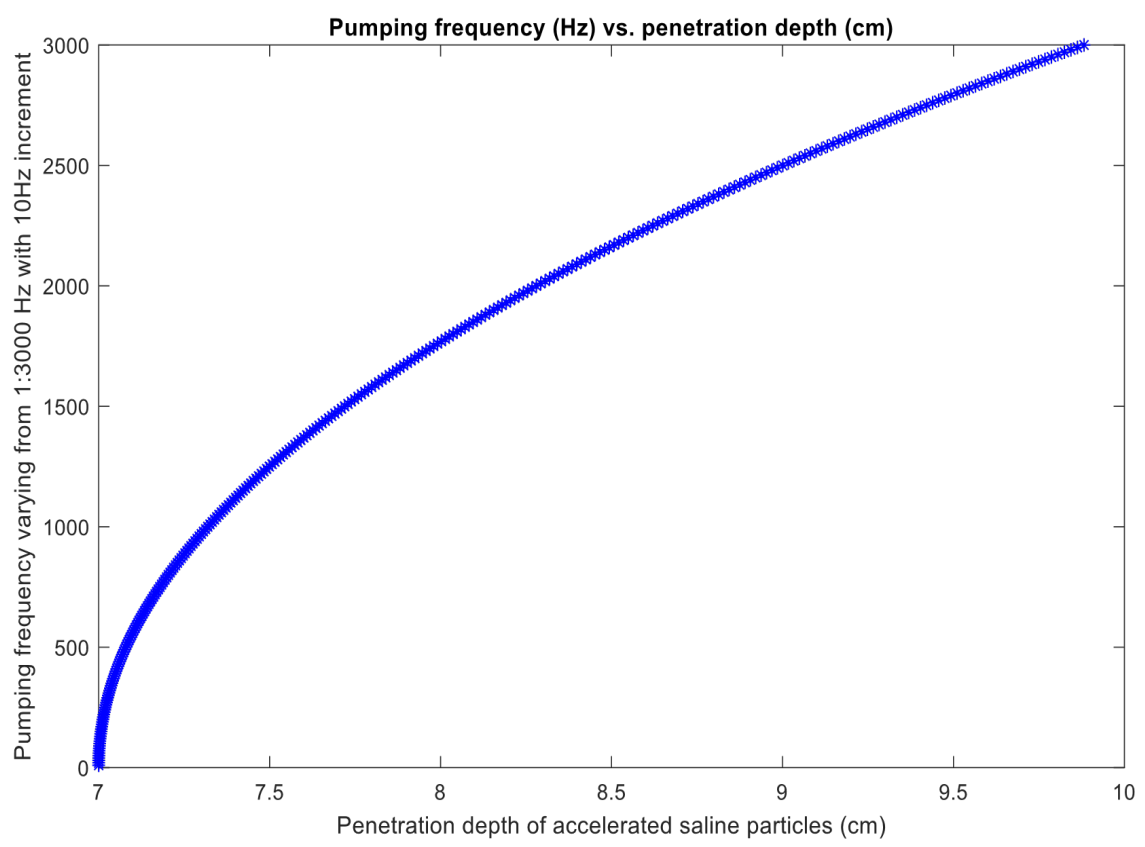

Figure 6. Curve produced while varying the pump frequency the penetration depth of the high speed particled solutions before reaching the original blood stream speed again.

be taken not to harm or break the blood vessels at such higher speeds and therefore speeds around $7 \mathrm{~cm} / \mathrm{s}$ seems safe as it is the original speed of the blood. For example, in order to reach a penetration depth of $7.5 \mathrm{~cm}$, a $1200 \mathrm{~Hz}$ pump is required and higher frequency pump is more expensive to build and for the prototype, we envisage to build a pump with low frequency of $300 \mathrm{~Hz}$ and investigate what happen in ongoing works.

\section{Discussions and Conclusions}

This is a preliminary and novel method investigating how accelerated particle saline solutions can be used to un-block partially blocked blood vessels. This method involves only a small invasive part in order to insert the needle (Figure A1) at the localized position ideally some centimetres away from the blockages or fat deposits in order for the accelerated particle solution can cleanse the walls and remove debris or fat deposits or blockages of the blood vessels (such as the veins). The proposed novel method so far based on a thorough research in this field employed the biological process which is involved in the inner ear which is the rippled movement of fluid that is created when one hears a sound in the ear. The rippled fluid movement is used to cleanse the vein, artery or blood vessel. The frequency of the pump is crucial in the depth of penetration of the accelerated fluid particles in cleaning the inner walls of the blood vessels. Results from this novel method are promising in terms of safe use of this catheter medical technique and in terms of the overall speed of blood flow even being bombarded by high-speed fluid particles that are governed by the pump frequency.

Now, in the near future, we will improve the model in terms of the texture and 
viscosity and ions actions that the saline solution can bring to this model in order to make it more efficient in curing blockages in the blood vessels (whether its arteries or veins). In order to achieve this, we will set up a physics laboratory where we will implement this novel method involving scientists, physicists and medical doctors.

\section{Conflicts of Interest}

The authors declare no conflicts of interest regarding the publication of this paper.

\section{References}

[1] Tan, T.S., Tan, K.H., Ng, H.P. and Loh, M.W. (2002) The Effects of Hypertonic Saline Solution (7.5\%) on Coagulation and Fibrinolysis: An In Vitro Assessment Using Thromboelastography. Anaesthesia, 57, 644-648. https://doi.org/10.1046/j.1365-2044.2002.02603.x

[2] Bellamy, M.L. and Frame, K. (Eds.) (1996) Neuroscience Laboratory and Classroom Activities. National Association of Biology Teachers and the Society for Neuroscience, 21-35.

[3] Camhi, J.M. (1984) Neuroethology: Nerve Cells and the Natural Behavior of Animals. Sinauer Associates, Inc., Sunderland.

[4] Kandel, E.R., Schwartz, J.H., and Jessell, T.M. (Eds.) (2000) Principles of Neural Science. 4th Edition, McGraw Hill Health Professions Division, New York.

[5] Shepherd, G.M. (1994) Neurobiology. 3rd Edition, Oxford University Press, Oxford, New York.

[6] Blood Flow, Blood Pressure, and Resistance. https://courses.lumenlearning.com/nemcc-ap/chapter/blood-flow-blood-pressure-a nd-resistance/

[7] Yamazaki, M., Kusano, K., Ishibashi, T., Kiuchi, M. and Koyama, K. (2015) Intravenous Infusion of $\mathrm{H}_{2}$-Saline Suppresses Oxidative Stress and Elevates Antioxidant Potential in Thoroughbred Horses after Racing Exercise. Scientific Reports, Article No. 15514. https://doi.org/10.1038/srep15514

[8] Chaui-Berlinck, J.G., Monteiro, L.H., Navas, C.A. and Bicudo, J.E. (2002) Temperature Effects on Energy Metabolism: A Dynamic System Analysis. Proceedings of the Royal Society Biological Science, 269, 15-19. https://doi.org/10.1098/rspb.2001.1845

[9] Primitives-Blender Manual. https://docs.blender.org/manual/en/latest/modeling/meshes/primitives.html

[10] Heinisch, M. (2015) Mechanical Resonators for Liquid Viscosity and Mass Density Sensing.

[11] Britannica, T. (Ed.) (2021) Conservation of Momentum. Encyclopedia Britannica. https://www.britannica.com/science/conservation-of-momentum

[12] Kleppner, D. and Kolenkow, R.J. (2010) An Introduction to Mechanics. Cambridge University Press, Cambridge, 112. https://doi.org/10.1017/CBO9780511794780 


\section{Appendix}

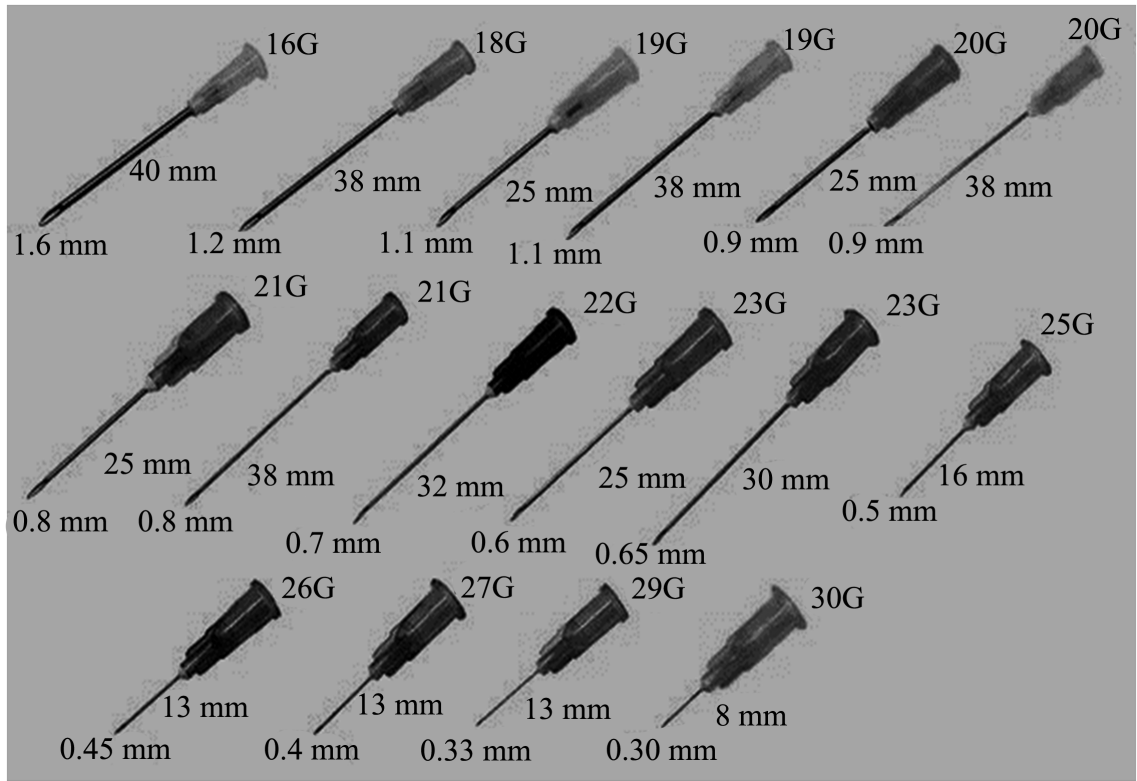

Figure A1. Types of needles and tips to demonstrate the one which is selected. 